\title{
Systemic-Lupus-Erythematosus-Related Acute Pancreatitis: A Cohort from South China
}

\author{
Yanlong Yang, ${ }^{1}$ Yujin Ye, ${ }^{1}$ Liuqin Liang, ${ }^{1}$ Tianfu $\mathrm{Wu},{ }^{2}$ Zhongping Zhan, ${ }^{1}$ \\ Xiuyan Yang, ${ }^{1}$ and Hanshi $\mathrm{Xu}^{1}$ \\ ${ }^{1}$ Department of Rheumatology, The 1st Affiliated Hospital, Sun Yat-Sen University, Guangzhou, Guangdong 510080, China \\ ${ }^{2}$ Department of Internal Medicine/Rheumatology, University of Texas Southwestern Medical Center, Dallas, TX 75390, USA
}

Correspondence should be addressed to Yujin Ye, graceyeyj@hotmail.com

Received 21 February 2012; Revised 26 April 2012; Accepted 29 April 2012

Academic Editor: Chaim Putterman

Copyright () 2012 Yanlong Yang et al. This is an open access article distributed under the Creative Commons Attribution License, which permits unrestricted use, distribution, and reproduction in any medium, provided the original work is properly cited.

\begin{abstract}
Acute pancreatitis (AP) is a rare but life-threatening complication of SLE. The current study evaluated the clinical characteristics and risk factors for the mortality of patients with SLE-related AP in a cohort of South China. Methods. Inpatient medical records of SLE-related AP were retrospectively reviewed. Results. 27 out of 4053 SLE patients were diagnosed as SLE-related AP, with an overall prevalence of $0.67 \%$, annual incidence of $0.56 \%$ and mortality of $37.04 \%$. SLE patients with AP presented with higher SLEDAI score $(21.70 \pm 10.32$ versus $16.17 \pm 7.51, P=0.03)$, more organ systems involvement $(5.70 \pm 1.56$ versus $3.96 \pm 1.15$, $P=0.001)$, and higher mortality (37.04\% versus $0, P=0.001)$, compared to patients without AP. Severe AP (SAP) patients had a significant higher mortality rate compared to mild AP (MAP) (75\% versus $21.05 \%, P=0.014)$. 16 SLE-related AP patients received intensive GC treatment, $75 \%$ of them exhibited favorable prognosis. Conclusion. SLE-related AP is rare but concomitant with high mortality in South Chinese people, especially in those SAP patients. Activity of SLE, multiple-organ systems involvement may attribute to the severity and mortality of AP. Appropriate glucocorticosteroid (GC) treatment leads to better prognosis in majority of SLE patients with AP.
\end{abstract}

\section{Introduction}

Systemic lupus erythematosus (SLE) is a chronic, autoimmune, inflammatory disease characterized by the presence of a plethora of autoantibodies, immune complex formation, and multiple organ system involvement. Gastrointestinal (GI) manifestations are common in SLE patients, but acute pancreatitis is rare [1-6]. It was reported that $19.2 \%-50 \%$ of SLE patients presented with gastrointestinal symptoms [711], whereas pancreatitis occurred in about $0.7 \%-8.2 \%$ of patients with SLE $[7,8,11,12]$ and the annual incidence was approximately $0.4-1.1 \%$ o [3-5]. Our knowledge about SLE-related acute pancreatitis (AP) is mostly based on individual case reports or small case series. Despite its rarity, AP can be a life-threatening complication of SLE if not treated appropriately. Prevalence of SLE is relatively high in Chinese people, which is $0.7 \sim 1 / 1000$ in comparison to $0.51 / 1000$ in United States [13]. But so far very few case reports on SLE-related AP in Chinese population have been published. The current study aims to clarify the clinical characteristics, severity, mortality, and outcome of SLErelated acute pancreatitis in south China.

\section{Materials and Methods}

A retrospective review of inpatient medical records between January 2000 and January 2012 was performed at the First Affiliated Hospital of Sun Yat-Sen University in South China. 4053 patients were classified as SLE during the past 12 years who fulfilled at least four of the American College of Rheumatology (ACR) revised classification criteria for SLE (1997) [14]. A diagnosis of acute pancreatitis (AP) was established by the presence of typical clinical symptoms (including abdominal pain, nausea, and vomiting) and confirmed by more than a three-fold elevation of serum amylase or lipase or evidence of imaging findings-computer tomography [CT] scan or ultrasonography (USG) [15]. Among these SLE patients, 27 were with dual simultaneous 
diagnosis of AP, and another 23 age- and gender-matched SLE patients without AP were randomly selected. Review of the clinical files of these 50 SLE patients was performed and data was extracted.

The SLE Disease Activity Index (SLEDAI) [16] was used to evaluate SLE activity during AP, and patients were defined as active SLE if the SLEDAI score was equal to or greater than 6. The Systemic Lupus International Collaborating Clinics/ACR (SLICC/ACR) damage index [17] was used to ascertain organ damage in SLE. The Atlanta criteria [18] were used to classify the severity of acute pancreatitis. Severe acute pancreatitis (SAP) was defined as the presence of at least three of Ranson's criteria and eight or more Acute Physiology and Chronic Health Evaluation II (APACHE II) score, or with the evidence of organ failure (systolic blood pressure $<90 \mathrm{mmHg}, \mathrm{PaO}_{2} \leq 60 \mathrm{mmHg}$ on room air, creatinine $>2 \mathrm{mg} / \mathrm{dL}$, gastrointestinal bleeding $>500 \mathrm{~mL} / 24 \mathrm{~h}$, DIC or severe hypocalcemia $\leq 7.5 \mathrm{mg} / \mathrm{dL}$ ) or local complications (i.e., pancreatic necrosis, abscess, or pseudocyst). The positivity of CT scan was defined as diffuse or segmental enlargement of the pancreas, illegibility of peripancreas fat, low/high density area in contrast, and peripancreas effusion [19]. The positivity of USG was defined as pancreatic enlargement, decreased echodensity, and possible fluid collections [20].

Demographic information including gender, age at SLE onset, duration between the onset of SLE and AP, history of alcohol consumption, gallstone, metabolic abnormalities (hypertriglyceridemia and hypercalcemia), clinical symptoms, laboratory findings, medications (especially corticosteroid, and immunosuppressive agents (ISA)) and outcome were documented. Acute pancreatitis related to mechanical obstruction (choledocholithiasis), toxic-metabolic etiologies (alcohol intake, drugs, hypercalcemia, or hypertriglyceridemia), infection, or trauma were ruled out in every case [21].

2.1. Statistical Analysis. Statistical analysis was done using the SPSS program 13.0 and Prism software version 5.0. The Mann-Whitney $U$ test was used for continuous variables and the chi-square or Fisher's exact test for categorical variables. Survival rates were estimated using the KaplanMeier method. A $P$ value $<0.05$ was considered statistically significant in all comparisons.

\section{Results}

3.1. Demographic and Clinical Characteristics of SLE-Related Acute Pancreatitis. 27 out of 4053 SLE patients were diagnosed as SLE-related AP during the past 12 years, with an overall prevalence of $0.67 \%$ and annual incidence of $0.56 \%$. One patient developed 2 episodes of pancreatitis and the other 26 patients had only one episode at the time of hospitalization. The demographic and clinical features of each SLE-related AP patient were shown in Table 1.

3.2. Comparison of Demographic and Clinical Features in SLE Patients with and without SLE-Related AP. The majority of patients $(92.59 \%, 25 / 27)$ were females and the mean age at SLE onset was $26.96 \pm 13.30$ years (ranged from 14 to 57 years). Time interval between the onset of SLE and AP ranged from 1 week to 20 years, and more than half of the patients $(51.85 \%, 14 / 27)$ developed AP within the first year of the onset of SLE. All these 27 patients were classified as active SLE with average SLEDAI score of $21.70 \pm 10.32$ at the onset of AP. The clinical features related to acute pancreatitis in these 27 SLE patients were nonspecific. Abdominal pain (92.59\%), fever (77.78\%) and nausea/vomiting (74.07\%), were the most frequent manifestations and other symptoms included diarrhea (44.44\%), loss of appetite (44.44\%) and GI tract hemorrhage (14.81\%).

Other organ system involvement was found in all SLErelated AP patients with an average number of $5.70 \pm$ 1.56 (ranged from 3 to 8 organs), including hematological system, kidney, liver, serositis, mucocutaneous involvement, respiratory system, arthritis, and central nervous system.

Clinical features and laboratory findings were compared between these two groups and the results were shown in Table 2. SLE patients with AP presented with higher SLEDAI score $(21.70 \pm 10.32$ versus $16.17 \pm 7.51, P=0.03)$, more organ system involvement $(5.70 \pm 1.56$ versus $3.96 \pm 1.15, P=$ $0.001)$, higher frequence of fever $(77.78 \%$ versus $39.13 \%, P=$ $0.006)$, hepatological and hematological disorders $(82.61 \%$ versus $34.78 \%, P=0.01 ; 100 \%$ versus $60.87 \%, P=0.001)$, serositis (62.96\% versus $26.09 \%, P=0.01)$, elevated CRP ( $81.82 \%$ versus $47.62 \%, P=0.02)$, positive anti-La antibody (33.33\% versus $0, P=0.003$ ), and higher mortality (37.04\% versus $0, P=0.001$ ) compared to SLE patients without AP.

\subsection{Comparison of Clinical Features between SAP and MAP} Patients. According to Atlanta criteria, 27 SLE-related AP patients were divided into SAP group (severe acute pancreatitis, $n=8,29.63 \%$ ) and MAP group (mild acute pancreatitis, $n=19,70.37 \%)$. The comparison of the demographic and clinical data between SAP and MAP patients as shown in Table 3. The results indicated that the age of onset of AP in SAP patients as significantly younger than MAP $(19.63 \pm 10.88$ versus $30.05 \pm 13.25, P=0.016)$. SAP patients presented with significantly higher mortality (75\% versus $21.05 \%, P=0.014$ ) and more abnormal hematologic findings (thrombocytopenia and leucopenia, $100 \%$ versus $52.63 \%, P=0.026 ; 87.5 \%$ versus $31.58 \%, P=$ 0.013 , resp.) compared to MAP. The Kaplan-Meier survival curves showed death rate within 30 days after onset of acute pancreatitis in SAP and MAP groups (Figure 1).

3.4. Comparison of Clinical Features between Pediatric-and Adult-Onset SLE-Related AP. SLE-related AP patients were divided into pediatric-onset group (under 18 years of age, $n=10)$ and adult-onset group $(n=17)$. Demographic and clinical characteristics were compared between these two groups. Pediatric-onset SLE-related AP had higher rate of severe AP (60\% versus $11.76 \%, P=0.014)$, higher serum amylase level (17.55 \pm 16.09 versus $6.53 \pm 5.42, P=0.007)$, lower percentage of positive anti-Ro antibody (25\% versus $84.62 \%, P=0.01)$, and lower rate of anti-La antibody 
TABLE 1: The demographic and clinical characteristics of each SLE patient with AP.

\begin{tabular}{|c|c|c|c|c|c|c|}
\hline Case & $\begin{array}{l}\text { Age at SLE onset } \\
(y)\end{array}$ & $\begin{array}{c}\text { Duration } \\
\text { between onset } \\
\text { of SLE and AP } \\
\text { (m) }\end{array}$ & $\begin{array}{l}\text { SLEDAI score at } \\
\text { onset of AP }\end{array}$ & $\begin{array}{l}\text { Number of } \\
\text { involved organs } \\
\text { concomitant } \\
\text { with AP }\end{array}$ & $\begin{array}{l}\text { GC treatment } \\
\text { after onset of AP }\end{array}$ & Outcome \\
\hline 1 & 23 & 0.5 & 9 & 5 & Increased dose & In remission \\
\hline 2 & 16 & 48 & 12 & 4 & Stop & In remission \\
\hline 3 & 18 & 12 & 14 & 6 & Increased dose & Died \\
\hline 4 & 22 & 72 & 14 & 7 & Increased dose & In remission \\
\hline 5 & 16 & 36 & 16 & 7 & Increased dose & In remission \\
\hline 6 & 57 & 24 & 17 & 4 & Increased dose & In remission \\
\hline 7 & 36 & 0.5 & 17 & 6 & Initial treatment & Died \\
\hline 8 & 48 & 180 & 18 & 7 & maintaining & Died \\
\hline 9 & 14 & 36 & 21 & 4 & maintaining & Died \\
\hline 10 & 14 & 12 & 23 & 7 & Increased dose & Died \\
\hline 11 & 19 & 2 & 23 & 6 & Increased dose & In remission \\
\hline 12 & 14 & 2 & 27 & 7 & Increased dose & Died \\
\hline 13 & 46 & 1 & 25 & 4 & Increased dose & In remission \\
\hline 14 & 22 & 0.25 & 18 & 3 & Decreased dose & In remission \\
\hline 15 & 51 & 240 & 18 & 6 & Decreased dose & In remission \\
\hline 16 & 42 & 4 & 19 & 7 & Increased dose & In remission \\
\hline 17 & 20 & 84 & 18 & 5 & Decreased dose & Died \\
\hline 18 & 39 & 24 & 13 & 5 & Increased dose & In remission \\
\hline 19 & 15 & 2 & 33 & 6 & Decreased dose & Died \\
\hline 20 & 15 & 36 & 41 & 7 & Increased dose & In remission \\
\hline 21 & 26 & 3 & 41 & 8 & Increased dose & Died \\
\hline 22 & 39 & 1 & 27 & 6 & Increased dose & In remission \\
\hline 23 & 20 & 72 & 10 & 3 & maintaining & In remission \\
\hline 24 & 36 & 12 & 38 & 6 & maintaining & Died \\
\hline 25 & 16 & 2 & 8 & 3 & Increased dose & In remission \\
\hline 26 & 30 & 48 & 47 & 8 & Increased dose & In remission \\
\hline 27 & 14 & 72 & 19 & 6 & maintaining & In remission \\
\hline
\end{tabular}

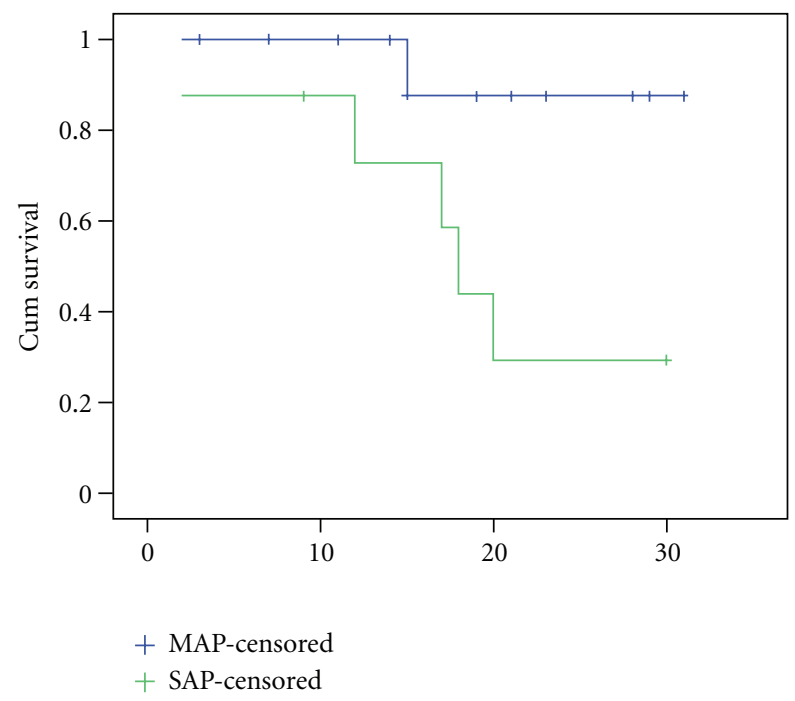

Figure 1: Kaplan-Meier survival curves for the time (days) from onset of SLE-related acute pancreatitis to death.
( 0 versus $53.85 \%, P=0.02$ ) compared to adult-onset SLErelated AP. However, the difference in mortality was not statistically significant between pediatric and adult patients (50\% versus $29.41 \%, P=0.26$ ).

3.5. Comparison of Clinical Features between Mortality and Nonmortality SLE Patients with AP. The risk factors for mortality were further analyzed. 27 SLE-related AP patients were divided into mortality group $(n=10)$ and nonmortality group $(n=17)$. The clinical manifestations were compared between these two groups and shown in Table 4. The mortality group had higher percentage of hypoalbuminemia (90\% versus $47.06 \%, P=0.031)$, hyperbilirubinemia $(40 \%$ versus $5.88 \%, P=0.047)$, hematuria ( $100 \%$ versus $41.18 \%$, $P=0.002)$, and granular casts $(70 \%$ versus $23.53 \%, P=$ $0.024)$ compared to nonmortality group. Severity of acute pancreatitis was the most powerful risk factor for mortality in SLE-related AP (OR 11.25, 95\% CI $(1.611,78.57)$ and $P=0.014$ ). 
TABLE 2: Comparison of demographic and clinical features in SLE patients with and without AP.

\begin{tabular}{|c|c|c|c|}
\hline & SLE with AP $(n=27)$ & SLE without AP $(n=23)$ & $P$ \\
\hline Female (\%) & $25(92.59 \%)$ & $20(86.96 \%)$ & 0.42 \\
\hline Age on SLE diagnosis (y) & $26.96 \pm 13.30$ & $28.39 \pm 9.98$ & 0.26 \\
\hline GCs dose (mg) & $61.19 \pm 37.63$ & $50.96 \pm 28.82$ & 0.18 \\
\hline SLEDAI score & $21.70 \pm 10.32$ & $16.17 \pm 7.51$ & 0.03 \\
\hline SLICC/ACR damage index & $1.19 \pm 0.92$ & $0.96 \pm 1.19$ & 0.11 \\
\hline Mortality & $10(37.04 \%)$ & 0 & 0.001 \\
\hline Fever $(\%)$ & $21(77.78 \%)$ & $9(39.13 \%)$ & 0.006 \\
\hline Neuropsychiatric (\%) & $7(25.93 \%)$ & $1(4.35 \%)$ & 0.042 \\
\hline Pulmonary $(\%)$ & $11(40.74 \%)$ & $5(21.74 \%)$ & 0.13 \\
\hline Articular (\%) & $16(59.26 \%)$ & $16(69.57 \%)$ & 0.32 \\
\hline Mucocutaneous involvement (\%) & $18(66.67 \%)$ & $16(69.57 \%)$ & 0.54 \\
\hline Renal (\%) & $24(88.89 \%)$ & $20(86.96 \%)$ & 0.59 \\
\hline Hepatological (\%) & $19(82.61 \%)$ & $8(34.78 \%)$ & 0.01 \\
\hline Hematological (\%) & $27(100.00 \%)$ & $14(60.87 \%)$ & 0.001 \\
\hline Serositis $(\%)$ & $17(62.96 \%)$ & $6(26.09 \%)$ & 0.01 \\
\hline Number of organs involved & $5.70 \pm 1.56$ & $3.96 \pm 1.15$ & 0.001 \\
\hline Positive anti-dsDNA (\%) & $24(88.89 \%)$ & $19(82.61 \%)$ & 0.41 \\
\hline Positive anti-Sm (\%) & $6 / 21(28.57 \%)$ & $10(43.48 \%)$ & 0.24 \\
\hline Positive anti-Ro (\%) & $13 / 21(61.90 \%)$ & $14(60.87 \%)$ & 0.60 \\
\hline Positive anti-La (\%) & $7 / 21(33.33 \%)$ & 0 & 0.003 \\
\hline Positive ACL-IgG (\%) & $4 / 21(19.05 \%)$ & $1 / 22(4.55 \%)$ & 0.168 \\
\hline Positive ACL-IgM (\%) & $4 / 21(19.05 \%)$ & $1 / 22(4.55 \%)$ & 0.16 \\
\hline Positive anti- $\beta_{2}$ GPI (\%) & $3 / 21(14.29 \%)$ & $2 / 22(9.09 \%)$ & 0.48 \\
\hline Low C3 (\%) & $26 / 26(100 \%)$ & $22(95.65 \%)$ & 0.47 \\
\hline Low C4 (\%) & $21 / 26(80.77 \%)$ & $20(86.96 \%)$ & 0.42 \\
\hline Elevated CRP (\%) & $18 / 22(81.82 \%)$ & $10 / 21(47.62 \%)$ & 0.02 \\
\hline
\end{tabular}

\section{Treatment and Outcome}

Among these 27 SLE-associated AP patients, 26 were on steroid treatment before the onset of AP and the average dosage of GCs was $61.19 \pm 37.63 \mathrm{mg} /$ day (ranged from $10 \mathrm{mg}$ /day to $120 \mathrm{mg}$ /day). AP was considered as the initial presentation of SLE in one patient (patient 7 in Table 1), and standard GC treatment started after diagnosis. Additional immunosuppressive agents (ISA) were also administrated in 22 patients before the onset of AP, including 18 on hydroxychloroquine, 2 azathioprine, 8 methotrexate, 5 cyclophosphamide, and 1 FK506. After the episodes of AP, oral medicines were stopped because of fasting. Methotrexate or cyclophosphamide were continuously prescribed in 5 patients but switched to I.V. injection. 1 patient developed recurrent episode of AP when increasing the dosage of GC for the relapse of SLE, and GC treatment was stopped (patient 2) after onset of AP. 25 patients were continuously treated by GCs and/or ISA during their episode of AP. 16 patients were given aggressive treatment of GCs and/or ISA (12 patients obtained clinical and laboratory improvement (75\%) and 4 died), 5 patients were treated with the maintenance dose of GCs and/or ISA (2 patients in remission (40\%) and 3 died), and 4 patients were treated with decreased dose of GCs because of fever and concerning of potential infections ( 2 patients in remission $(50 \%)$ and 2 died) (The results showed in Figure 2). Totally, 10 patients died and the overall mortality rate was $37.04 \%(10 / 27)$.

\section{Discussion}

SLE-related AP is relatively rare compared to other organ injury involved in lupus. The incidence of clinical AP associated with SLE varies from 0.7 to $4 \%[5,8,12,22]$, with the annual incidence of $0.4-1.1 \%$ o $[3,4]$. Most previous studies on this issue were individual case reports or small case series. So far, the Hopkins lupus cohort [12] reported the largest case series with 63 SLE-attribute pancreatitis out of 1740 SLE patients (3.5\%), and a Taiwan series reported 40 out of 2976 SLE patients (1.34\%). This study was the first report of the SLE-related AP in south China. In current cohort, 27 out of 4053 SLE patients were diagnosed as SLE-related AP, with the prevalence of $0.67 \%$, and annual incidence of $0.56 \%$, which is comparable with the findings of previous literatures [3-5, 8, 12, 22].

The pathogenic mechanism of SLE-related AP is very complex and multifactors. Vascular damage (including vasculitis, intimal thickening, immune complex deposition, occlusion of arteries, and arterioles), autoantibody production, abnormal cellular immune response, and drug toxicity may be responsible for the development of pancreatitis [8]. 
TABLE 3: Comparison of demographic and clinical characteristics between SLE-related severe acute pancreatitis (SAP) and mild acute pancreatitis (MAP).

\begin{tabular}{|c|c|c|c|}
\hline & $\operatorname{SAP}(n=8)$ & $\operatorname{MAP}(n=19)$ & $P$ value \\
\hline \multicolumn{4}{|l|}{ Demographic characteristics } \\
\hline Female & $7(87.50 \%)$ & $18(94.74 \%)$ & 0.513 \\
\hline Age of onset AP (y) & $19.63 \pm 10.88$ & $30.05 \pm 13.25$ & 0.016 \\
\hline Interval between onset of SLE and AP (m) & $23.38 \pm 28.25$ & $44.36 \pm 64.73$ & 0.822 \\
\hline Early AP $(\leqslant 1$ year $)$ & $5(62.50 \%)$ & $9(47.37 \%)$ & 0.678 \\
\hline SLEDAI score at onset of AP & $22.13 \pm 6.24$ & $21.53 \pm 11.77$ & 0.44 \\
\hline SLICC/ACR damage index & $1.25 \pm 0.89$ & $1.16 \pm 0.96$ & 0.854 \\
\hline Number of organs involved & $5.75 \pm 1.28$ & $5.68 \pm 1.70$ & 0.893 \\
\hline Intensive therapy of GC/ISA & $5(62.50 \%)$ & $11(57.89 \%)$ & 1 \\
\hline Mortality & $6(75 \%)$ & $4(21.05 \%)$ & 0.014 \\
\hline \multicolumn{4}{|l|}{ Clinical characteristics } \\
\hline Fever & $8(100.00 \%)$ & $13(68.42 \%)$ & 0.136 \\
\hline Mucocutaneous involvement & $6(75.00 \%)$ & $12(63.16 \%)$ & 0.676 \\
\hline Articular involvement & $3(37.50 \%)$ & $10(52.63 \%)$ & 0.678 \\
\hline Serositis & $5(62.50 \%)$ & $10(52.63 \%)$ & 0.696 \\
\hline Neuropsychiatric involvement & $2(25.00 \%)$ & $5(26.32 \%)$ & 1 \\
\hline Renal involvement & $7(87.50 \%)$ & $15(78.95 \%)$ & 1.0 \\
\hline \multicolumn{4}{|l|}{ Laboratory findings } \\
\hline Serum amylase* & $18.09 \pm 18.15$ & $7.46 \pm 5.88$ & 0.077 \\
\hline Serum lipase* & $8.53 \pm 3.14$ & $7.63 \pm 5.45$ & 0.616 \\
\hline Elevated serum transaminase & $7(87.5 \%)$ & $11(57.89 \%)$ & 0.201 \\
\hline Thrombocytopenia & $8(100 \%)$ & $10(52.63 \%)$ & 0.026 \\
\hline Leucopenia & $7(87.50 \%)$ & $6(31.58 \%)$ & 0.013 \\
\hline Positive anti-dsDNA & $8(100.00 \%)$ & $16(84.21 \%)$ & 0.532 \\
\hline Positive anti-Sm & $1 / 7(14.29 \%)$ & $5 / 14(35.71 \%)$ & 0.613 \\
\hline Low C3 & $8 / 8(100.00 \%)$ & $18 / 18(100.00 \%)$ & 1 \\
\hline Low C4 & $7 / 8(87.50 \%)$ & $14 / 18(77.78 \%)$ & 1 \\
\hline Anti-Ro & $2 / 7(28.57 \%)$ & $11 / 14(78.57 \%)$ & 0.056 \\
\hline Anti-La & $1 / 7(14.29 \%)$ & $6 / 14(42.86 \%)$ & 0.337 \\
\hline
\end{tabular}

${ }^{*}$ Times in excess of the upper limit of normal (ULN).

In the current cohort, more than half patients (51.85\%) developed acute pancreatitis within 1 year of the onset of SLE, and all 27 patients were active SLE with dramatically elevated SLEDAI scores and other simultaneous SLE manifestations, especially the hematologic and renal involvement. SLE patients with AP presented with higher SLEDAI scores compared to patients without AP. Previous studies [3, 4, 22, 23] also demonstrated that episodes of SLE-related pancreatitis significantly increased in the active SLE group. AP was considered as one of the clinical features of active SLE and was associated with the activity of the disease itself. These results indicated that SLE itself can be the primary etiologic factor or cofactor predisposing to AP.

SLICC/ACR damage index score represents disease burden in SLE patients. It was significantly higher in SLE patients with pancreatitis compared to SLE patients without pancreatitis in Hopkins cohort [12]. Although SLE-related AP had more organ system involvement in current study, the damage index score was low, and there was no significant difference between SLE patients with and without AP $(1.19 \pm$ 0.92 versus $0.96 \pm 1.19, P=0.11)$. The reason of the low-damage index score might lie in the relatively younger onset age, shorter duration of disease, and less-chronic organ damage.

Our study found that pediatric-onset AP tended to be more severe compared to adult-onset AP. SAP group had significant higher prevalence of thrombocytopenia and leucopenia than MAP group. Mortality patients has higher rate of hypoalbuminemia, hematuria, granular casts, and hyperbilirubinemia than nonmortality group, which indicated that multiple organ systems involvement, especially hematological, renal, and liver injury in SLE patients might be the major causes due to the severity and mortality of AP. In general population, the mortality rate of AP is about $3.8 \% \sim 10 \%$ [24-27]. Approximately $15 \sim 20 \%$ of all AP cases were SAP which accounted for a mortality rate of $16.3 \%$ $30 \%$ [27-29]. SLE-related AP patients had much higher mortality. Wang et al. [23] reported that the mortality rate was $27.5 \%$ in all SLE-related AP and $78.57 \%$ in SAP. Richer et al. [30] reported that $57 \%$ of childhood-onset lupus with pancreatitis developed SAP with the mortality of $45 \%$. In our cohort, the overall mortality rate of SLE-related AP was 
TABLE 4: Comparison of demographic and clinical characteristics between mortality and non-mortality group.

\begin{tabular}{|c|c|c|c|}
\hline & $\begin{array}{c}\text { Mortality } \\
(n=10)\end{array}$ & $\begin{array}{l}\text { Non-mortality } \\
\quad(n=17)\end{array}$ & $P$ value \\
\hline \multicolumn{4}{|l|}{ Demographic characteristics } \\
\hline Female & $10(100 \%)$ & $15(88.24 \%)$ & 0.387 \\
\hline Age of onset AP (y) & $24.10 \pm 12.02$ & $28.65 \pm 14.07$ & 0.123 \\
\hline SLEDAI score at onset of AP & $25.00 \pm 9.40$ & $19.76 \pm 10.61$ & 0.065 \\
\hline Number of organs involved & $6.20 \pm 1.14$ & $5.41 \pm 1.73$ & 0.167 \\
\hline Intensive therapy of GC/ISA & $4(40 \%)$ & $12(70.59 \%)$ & 0.124 \\
\hline \multicolumn{4}{|l|}{ Clinical characteristics } \\
\hline Fever & $10(100 \%)$ & $11(64.71 \%)$ & 0.042 \\
\hline Mucocutaneous involvement & $6(60 \%)$ & $12(70.59 \%)$ & 0.439 \\
\hline Articular involvement & $6(60 \%)$ & $7(41.18 \%)$ & 0.293 \\
\hline Serositis & $7(70 \%)$ & $8(47.06 \%)$ & 0.226 \\
\hline Neuropsychiatric involvement & $4(40 \%)$ & $3(17.65 \%)$ & 0.204 \\
\hline \multicolumn{4}{|l|}{ Laboratory findings } \\
\hline Serum amylase* & $14.79 \pm 17.34$ & $8.15 \pm 6.03$ & 0.241 \\
\hline Serum lipase* & $6.46 \pm 3.51$ & $8.20 \pm 5.58$ & 0.368 \\
\hline Elevated serum transaminase & $8(80 \%)$ & $10(58.82 \%)$ & 0.244 \\
\hline Hypoalbuminemia & $9(90 \%)$ & $8(47.06 \%)$ & 0.031 \\
\hline Proteinuria & $10(100 \%)$ & $12(70.59 \%)$ & 0.077 \\
\hline Hematuria & $10(100 \%)$ & $7(41.18 \%)$ & 0.002 \\
\hline Granular casts & $7(70 \%)$ & $4(23.53 \%)$ & 0.024 \\
\hline Hyperbilirubinemia & $4(40 \%)$ & $1(5.88 \%)$ & 0.047 \\
\hline Positive anti-dsDNA & $10(100 \%)$ & $14(82.35 \%)$ & 0.232 \\
\hline Positive anti-Sm & $2 / 8(25 \%)$ & $4 / 13(30.77 \%)$ & 0.59 \\
\hline Low C3 & 9/9 (100\%) & $17 / 17(100 \%)$ & 1.0 \\
\hline Low C4 & $7 / 9(77.78 \%)$ & $14 / 17(82.35 \%)$ & 0.58 \\
\hline Anti-Ro & $3 / 8(37.5 \%)$ & $10 / 13(76.92 \%)$ & 0.09 \\
\hline Anti-La & $1 / 8(12.5 \%)$ & $6 / 13(46.15 \%)$ & 0.133 \\
\hline
\end{tabular}

${ }^{*}$ Times in excess of the upper limit of normal (ULN).

$37.04 \%$ compared to 0 in SLE patients without AP $(P=$ 0.001 ), and mortality rate in SAP was $75 \%$. The severity of AP might be the most important risk factor for the mortality of SLE-related AP patients (OR 11.25, 95\% CI $(1.611,78.57)$, and $P=0.014$ ).

In accordance with other literatures, the manifestations of SLE-related AP in this cohort were nonspecific and similar to non-SLE acute pancreatitis. Abdominal pain $(92.59 \%)$, fever $(77.78 \%)$, and nausea/vomiting $(74.07 \%)$ were the most common symptoms. These symptoms could also be attributed to other gastrointestinal diseases or adverse reactions of medication and may lead to misdiagnosis in general practice. It was reported that the rate of misdiagnosis of AP in SLE was up to $88.6 \%$ [31]. Delayed diagnosis and improper treatment may contribute to unfavorable prognosis, even lifethreatening [32]. Likewise, the mortality rate of the Hopkins Lupus Cohort (3\%) was considerably lower than average of other reported studies due to close monitoring, early diagnosis, and treatment [12]. So, AP should be paid more attention in any SLE patient with abdominal pain when mechanical obstruction or toxic-metabolic etiologies, infection, or trauma were ruled out.
Some immunosuppressants, such as corticosteroids, azathioprine, and cyclosporine have been implicated to cause pancreatitis in several case reports. Only 2 patients in our study took azathioprine but the medication was discontinued after the onset of AP. The current study couldn't verify the relationship between azathioprine and acute pancreatitis in SLE patients. There is still a controversy over steroid treatment in SLE-related AP. Increasingly accumulated evidence showed that steroids do not trigger acute pancreatitis or cause increased mortality on AP [22, 33, 34], but instead, they have a possible therapeutic effect on SLE-related pancreatitis [5, 35-37]. In Hopkins cohort, appropriate treatment with corticosteroids added a survival benefit in SLE-related AP. In current study, 16 SLE-related AP patients received intensive GC and/or ISA treatment, and $75 \%$ of them exhibited favorable prognosis.

In summary, SLE-related acute pancreatitis is rare but with high-mortality rate, which is even higher in those severe acute pancreatitis with multiple organ system involvement. Activity of SLE, hematological system, renal, and liver injury in SLE patients may attribute to the mortality of AP. Early diagnosis of acute pancreatitis in SLE patients, especially 


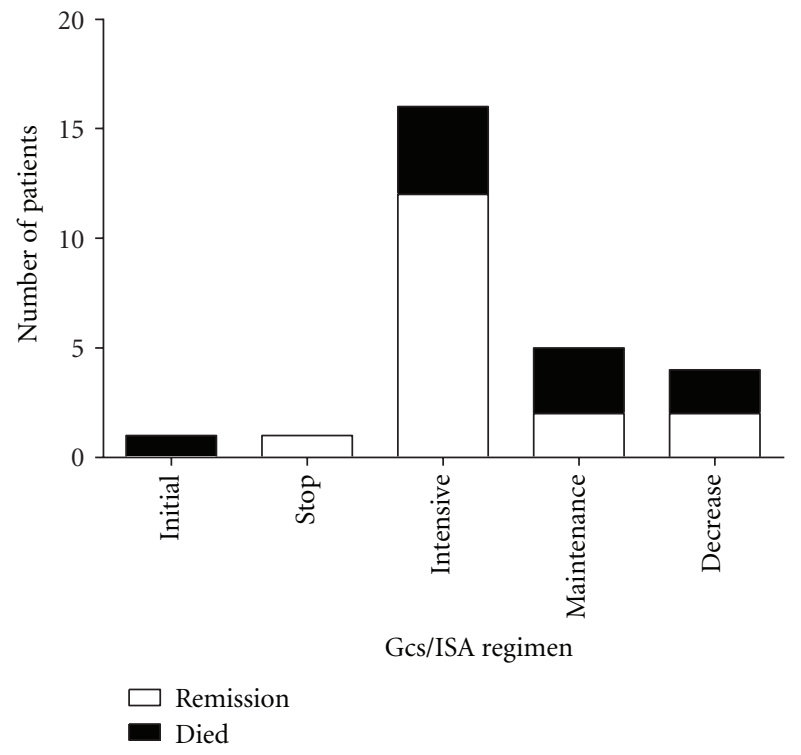

FIgure 2: Treatment regimen and outcome of the SLE-related AP.

those with abdominal pain, and appropriate glucocorticosteroid treatment is beneficial for a better therapeutic outcome in the majority of patients.

\section{Abbreviations}

AP: Acute pancreatitis

SAP: Severe acute pancreatitis

MAP: Mild acute pancreatitis

GC: Glucocorticosteroid

ISA: Immunosuppressive agents.

\section{Acknowledgments}

This work was supported by the Guangdong Provincial Science and Technology Funds, China (2009B030801098, 2011B050300009, 2008B080703015).

\section{References}

[1] D. Xu, H. Yang, C. C. Lai et al., "Clinical analysis of systemic lupus erythematosus with gastrointestinal manifestations," Lupus, vol. 19, no. 7, pp. 866-869, 2010.

[2] D. S. Myung, T. J. Kim, S. J. Lee et al., "Lupus-associated pancreatitis complicated by pancreatic pseudocyst and central nervous system vasculitis," Lupus, vol. 18, no. 1, pp. 74-77, 2009.

[3] G. S. Breuer, A. Baer, D. Dahan, and G. Nesher, "Lupusassociated pancreatitis," Autoimmunity Reviews, vol. 5, no. 5, pp. 314-318, 2006.

[4] G. Nesher, G. S. Breuer, K. Temprano et al., "Lupus-associated pancreatitis," Seminars in Arthritis and Rheumatism, vol. 35, no. 4, pp. 260-267, 2006.

[5] C. T. Derk and R. J. DeHoratius, "Systemic lupus erythematosus and acute pancreatitis: a case series," Clinical Rheumatology, vol. 23, no. 2, pp. 147-151, 2004.

[6] L. Essaadouni, E. Samar, and K. Krati, "Pancreatitis as initial manifestation of systemic lupus erythematosus," Lupus, vol. 19, no. 7, pp. 884-887, 2010.
[7] G. S. Firestein, R. C. Budd, E. D. Harris Jr, I. B. McInnes, S. Ruddy, and J. S. Sergent, Kelley's Textbook of Rheumatology, W. B. Saunders Company, Philadelphia, Pa, USA, 8th edition, 2008.

[8] J. L. Noia, F. M. García, S. S. Ríos, J. Iglesias García, and J. E. Domínguez Muñoz, "Pancreatitis and systemic lupus erythematosus," Revista Espanola de Enfermedades Digestivas, vol. 101, no. 8, pp. 571-579, 2009.

[9] F. Wang, N. S. Wang, B. H. Zhao, and L. Q. Tang, "Acute pancreatitis as an initial symptom of systemic lupus erythematosus: a case report and review of the literature," World Journal of Gastroenterology, vol. 11, no. 30, pp. 4766-4768, 2005.

[10] M. S. Al-Hakeem and M. A. McMillen, "Evaluation of abdominal pain in systemic lupus erythematosus," American Journal of Surgery, vol. 176, no. 3, pp. 291-294, 1998.

[11] J. L. Huang, C. C. Huang, C. Y. Chen, and I. J. Hung, "Acute pancreatitis: an early manifestation of systemic lupus erythematosus," Pediatric Emergency Care, vol. 10, no. 5, pp. 291-293, 1994.

[12] A. Makol and M. Petri, "Pancreatitis in systemic lupus erythematosus: frequency and associated factors-a review of the Hopkins Lupus Cohort," Journal of Rheumatology, vol. 37, no. 2, pp. 341-345, 2010.

[13] P. Forabosco, J. D. Gorman, C. Cleveland et al., "Metaanalysis of genome-wide linkage studies of systemic lupus erythematosus," Genes and Immunity, vol. 7, no. 7, pp. 609614, 2006.

[14] M. C. Hochberg, "Updating the American college of rheumatology revised criteria for the classification of systemic lupus erythematosus," Arthritis and Rheumatism, vol. 40, no. 9, p. $1725,1997$.

[15] J. H. C. Ranson and P. Shamamian, "Diagnostic standards for acute pancreatitis," World Journal of Surgery, vol. 21, no. 2, pp. 136-142, 1997.

[16] C. Bombardier, D. D. Gladman, M. B. Urowitz, D. Caron, and C. H. Chang, "Derivation of the SLEDAI. A disease activity index for lupus patients. The committee on prognosis studies in SLE," Arthritis \& Rheumatism, vol. 35, no. 6, pp. 630-640, 1992.

[17] D. Gladman, E. Ginzler, C. Goldsmith et al., "The development and initial validation of the Systemic Lupus International Collaborating Clinics/American College of Rheumatology damage index for systemic lupus erythematosus," Arthritis and Rheumatism, vol. 39, no. 3, pp. 363-369, 1996.

[18] E. L. Bradley and C. F. Frey, "A clinically based classification system for acute pancreatitis: summary of the International Symposium on Acute Pancreatitis, Atlanta, Ga, September 11 through 13, 1992," Archives of Surgery, vol. 128, no. 5, pp. 586590, 1993.

[19] W. Silverstein, M. B. Isikoff, M. C. Hill, and J. Barkin, "Diagnostic imaging of acute pancreatitis: prospective study using CT and sonography," American Journal of Roentgenology, vol. 137, no. 3, pp. 497-502, 1981.

[20] D. A. Sarti and W. King, "The ultrasonic findings in inflammatory pancreatic disease," Seminars in Ultrasound, vol. 1, no. 3, pp. 178-191, 1980.

[21] D. Whitcomb, "Acute pancreatitis," The New England Journal of Medicine, vol. 354, pp. 2142-2150, 2006.

[22] V. Pascual-Ramos, A. Duarte-Rojo, A. R. Villa et al., "Systemic lupus erythematosus as a cause and prognostic factor of acute pancreatitis," Journal of Rheumatology, vol. 31, no. 4, pp. 707$712,2004$. 
[23] C. H. Wang, T. C. Yao, Y. L. Huang, L. S. Ou, K. W. Yeh, and J. L. Huang, "Acute pancreatitis in pediatric and adult-onset systemic lupus erythematosus: a comparison and review of the literature," Lupus, vol. 20, no. 5, pp. 443-452, 2011.

[24] P. J. Fagenholz, C. F. D. Castillo, N. S. Harris, A. J. Pelletier, and C. A. Camargo Jr, "Increasing United States hospital admissions for acute pancreatitis, 1988-2003," Annals of Epidemiology, vol. 17, no. 7, pp. 491-e1, 2007.

[25] G. Cavallini, L. Frulloni, C. Bassi et al., "Prospective multicentre survey on acute pancreatitis in Italy (ProInf-AISP): results on 1005 patients," Digestive and Liver Disease, vol. 36, no. 3, pp. 205-211, 2004.

[26] D. V. Mann, M. J. Hershman, R. Hittinger, and G. Glazer, "Multicentre audit of death from acute pancreatitis," British Journal of Surgery, vol. 81, no. 6, pp. 890-893, 1994.

[27] C. Y. Fu, C. N. Yeh, J. T. Hsu, Y. Y. Jan, and T. L. Hwang, "Timing of mortality in severe acute pancreatitis: experience from 643 patients," World Journal of Gastroenterology, vol. 13, no. 13, pp. 1966-1969, 2007.

[28] T. H. Baron and D. E. Morgan, "Acute necrotizing pancreatitis," New England Journal of Medicine, vol. 340, no. 18, pp. 1412-1417, 1999.

[29] R. Isenmann, B. Rau, and H. G. Beger, "Bacterial infection and extent of necrosis are determinants of organ failure in patients with acute necrotizing pancreatitis," British Journal of Surgery, vol. 86, no. 8, pp. 1020-1024, 1999.

[30] O. Richer, T. Ulinski, I. Lemelle et al., "Abdominal manifestations in childhood-onset systemic lupus erythematosus," Annals of the Rheumatic Diseases, vol. 66, no. 2, pp. 174-178, 2007.

[31] X. Zhang, X. J. Zeng, Y. Dong et al., "Digestive system involvement in systemic lupus erythematosus," Chinese Journal of Digestive Diseases, vol. 19, no. 1, pp. 42-44, 1999.

[32] U. R. Nwaneri, C. O. Callender, and J. E. Stevens, "Lupus pancreatitis," Journal of the National Medical Association, vol. 87, no. 8, pp. 575-576, 1995.

[33] J. C. Reynolds, R. D. Inman, and R. P. Kimberly, "Acute pancreatitis in systemic lupus erythematosus: report of twenty cases and a review of the literature," Medicine, vol. 61, no. 1, pp. 25-32, 1982.

[34] W. M. Steinberg and J. H. Lewis, "Steroid-induced pancreatitis: does it really exist?” Gastroenterology, vol. 81, no. 4, pp. 799-808, 1981.

[35] S. Saab, M. P. Corr, and M. H. Weisman, "Corticosteroids and systemic lupus erythematosus pancreatitis: a case series," Journal of Rheumatology, vol. 25, no. 4, pp. 801-806, 1998.

[36] D. Kapoor, E. Mendez, and L. R. Espinoza, "Corticosteroids and SLE pancreatitis," Journal of Rheumatology, vol. 26, no. 4, pp. 1011-1012, 1999.

[37] M. Petri, "Pancreatitis in systemic lupus erythematosus: still in search of a mechanism," Journal of Rheumatology, vol. 19, no. 7, pp. 1014-1016, 1992. 


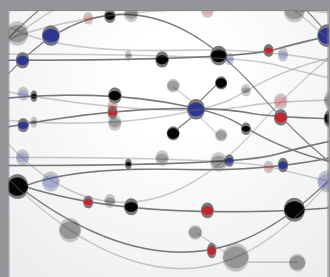

The Scientific World Journal
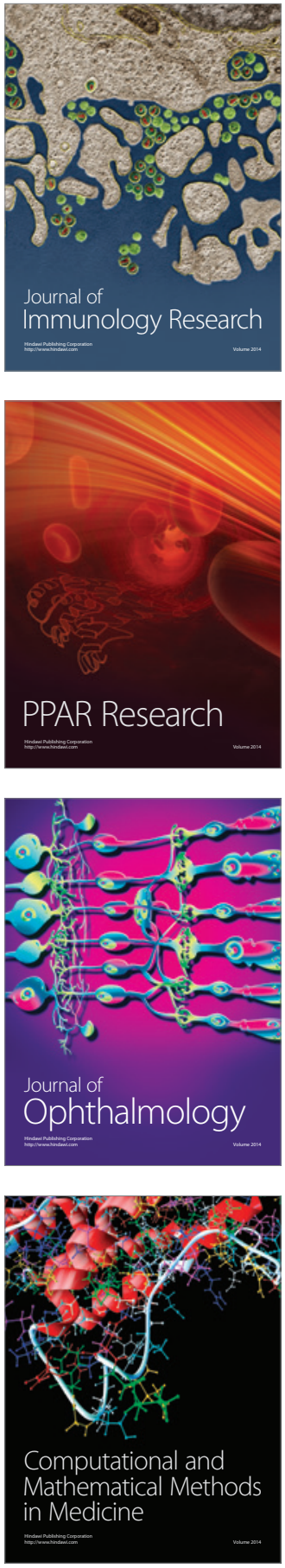

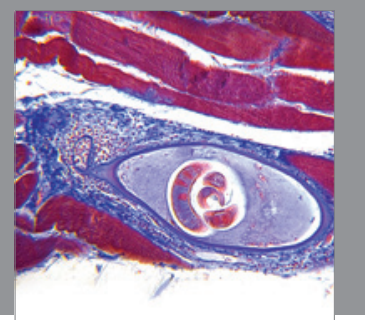

Gastroenterology

Research and Practice
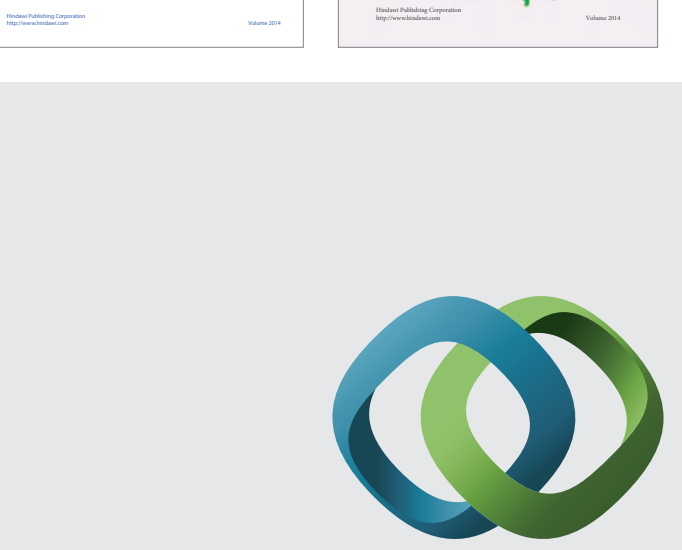

\section{Hindawi}

Submit your manuscripts at

http://www.hindawi.com
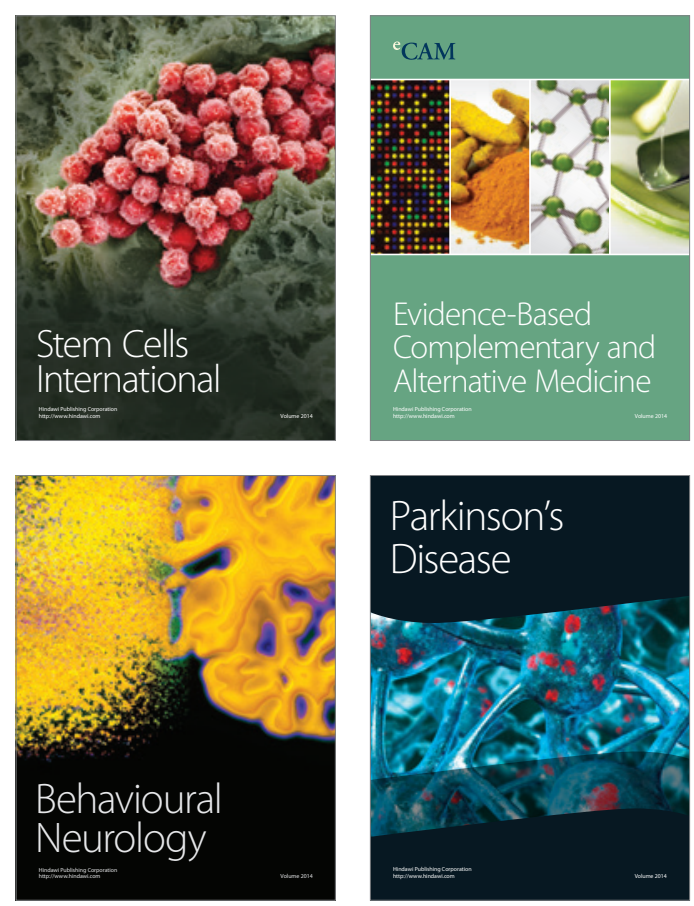

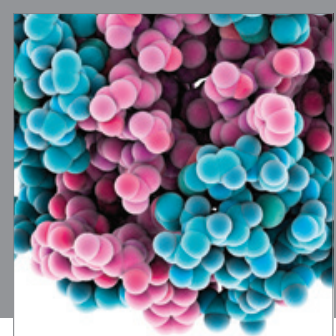

Journal of
Diabetes Research

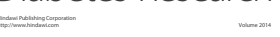

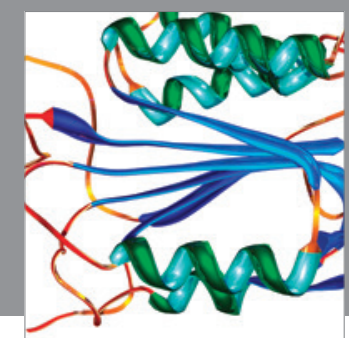

Disease Markers
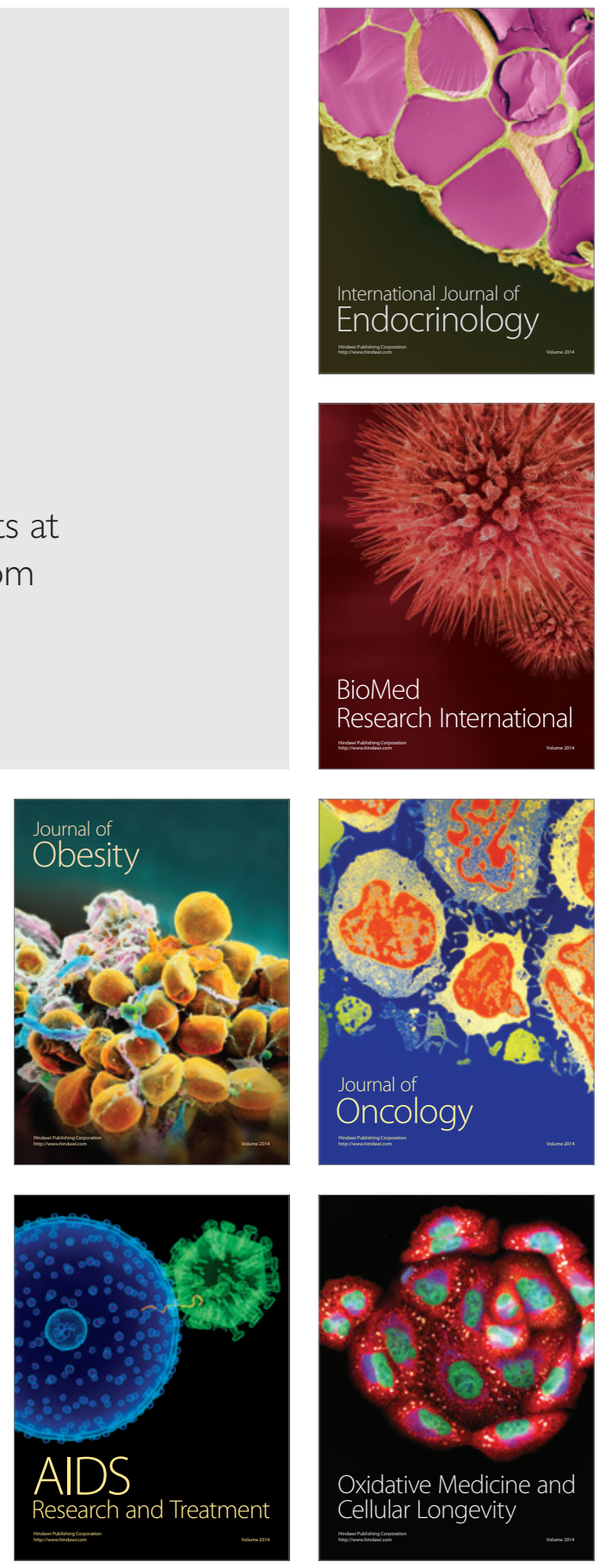